\title{
Nasality Changes With Age in Normal Korean-Speaking Adults
}

\author{
Jun $\mathrm{Xu}^{1,2} \cdot$ Young-Ae Kang ${ }^{1} \cdot$ Soo-Kyoung Park ${ }^{1}$ Young Hoon Yoon ${ }^{1} \cdot$ Shang Jie Bai ${ }^{2}$ Yong De $\mathrm{Jin}^{2} \cdot$ Yong Min Kim $^{1}$ \\ Ki-Sang Rha ${ }^{1}$
}

${ }^{1}$ Department of Otorhinolaryngology-Head and Neck Surgery, Chungnam National University School of Medicine, Daejeon, Korea; ${ }^{2}$ Department of Otorhinolaryngology-Head and Neck Surgery, Yanbian University Hospital, Yanji, China

Objectives. This study was performed to investigate the effects of aging on nasality and the influence of age-related changes in nasal cavity volume and nasal patency on nasality.

Methods. A total of 180 healthy Korean-speaking adult volunteers, who had no nasal or voice-related complaints, were enrolled in this study. Nasometry, acoustic rhinometry, and rhinomanometry were performed to obtain the nasalance score, nasal cavity volume, and nasal resistance, respectively. Changes in these parameters with age were analyzed.

Results. Nasal cavity volume increased significantly, and nasal resistance decreased significantly, with age. The nasalance scores for the nasal passage and oronasal passage decreased significantly with age, while there were no age-related changes in nasalance scores for the oral passage.

Conclusion. Nasalance scores for the passages containing nasal consonants decreased with age although significant increases were observed in nasal cavity volume and nasal patency with age. Therefore, the age-related decreases in nasalance scores may result from factors other than changes in the nasal cavity.

Keywords. Voice Quality; Nasal Cavity; Acoustic Rhinometry; Rhinomanometry; Aging; Adult

\section{INTRODUCTION}

Nasal speech sounds are produced when the oral and nasal cavities are connected [1,2]. Therefore, any changes in volume or geometry of the nasal cavity, relative impedance of the nasal and oral cavities, and velopharyngeal function can affect nasal resonance and result in a perceptual change in quality of the nasal sound, called nasality [3].

To assess nasal resonance problems, reference nasal resonance scores for normal subjects are needed. Measurement of nasalance with a nasometer provides a useful measure of nasality.

\footnotetext{
- Received May 18, 2018

Revised July 12, 2018

Accepted August 3, 2018

- Corresponding author: Ki-Sang Rha

Department of Otorhinolaryngology-Head and Neck Surgery, Chungnam

National University School of Medicine, 266 Munhwa-ro, Jung-gu, Daejeon 35015, Korea

Tel: +82-42-280-7698, Fax: +82-42-253-4059

E-mail: ksrha@cnu.ac.kr
}

There have been a number of studies of nasalance scores that serve as references for nasometric assessment [2,4-10]. However, it has been reported that age, sex, dialect, race, and language may affect normative nasalance scores [5,6].

Aging is a basic physiological process affecting structural and functional aspects of all parts of the body, including the nasal cavity and velopharynx, and may therefore influence nasality. However, previous studies on the effects of age on nasalance scores have yielded inconsistent results, and all were comparative studies between children and adults or between children of different ages $[2,4,6-8,10]$. To date, there have been no studies comparing nasalance scores across adults categorized into different age groups.

The present study was performed to obtain normative nasalance data from normal Korean-speaking adults, including elderly subjects, and to investigate the possible influence of agerelated changes in the nasal cavity (nasal cavity volume and nasal resistance) on nasalance scores.

Copyright $\odot 2019$ by Korean Society of Otorhinolaryngology-Head and Neck Surgery.

This is an open-access article distributed under the terms of the Creative Commons Attribution Non-Commercial License (http://creativecommons.org/licenses/by-nc/4.0)

which permits unrestricted non-commercial use, distribution, and reproduction in any medium, provided the original work is properly cited. 


\section{MATERIALS AND METHODS}

\section{Subjects}

A total of 180 native healthy adult Korean speakers (90 men and 90 women) living in Daejeon, South Korea were enrolled in this cross-sectional study. The subjects ranged in age from 20 to 70 years and were divided into five groups: $20-29,30-39,40$ $49,50-59$, and over 60 years. Each age group consisted of 36 subjects (18 men and 18 women). All of the participants had normal hearing and speech for their age. None had a history of velopharyngeal or sinonasal surgery. Subjects with any disorder leading to nasal obstruction, such as colds, allergic rhinitis, sinusitis, nasal polyposis, or severe septal deviation were excluded.

The study protocol was approved by the Institutional Review Board of Chungnam National University Hospital. Informed consent was obtained from each participant before enrollment into the study.

\section{Measurements}

Nasometry, acoustic rhinometry (AR), and rhinomanometry were performed to determine the nasalance score, nasal cavity volume, and nasal resistance, respectively, using appropriate instruments. All of the instruments were installed in a sound-treated room, and were calibrated and operated according to the respective manufacturer's instructions. Subjects rested in the study room for 20 minutes before the test to acclimatize to the environment. No topical decongestant, which can lead to changes in measurement data, was administered to any subject.

\section{Nasometry}

Nasalance scores were obtained using a Nasometer II 3.4 system (model 6450; KayPENTAX, Lincoln Park, NJ, USA). Subjects were seated on a chair with a headset, maintaining the sound separator palate in the horizontal plane between the lips and the nose. They were asked to read three different passages in Korean designed by Park et al. [2], i.e., an oral passage, an oronasal passage, and a nasal passage, consisting of 33, 37, and 24 Korean syllables, respectively. The oral passage was devoid of nasal consonants to allow assessment of hypernasality. The oronasal passage consisted of a mixture of oral and nasal consonants $(32.6 \%)$, based on the phonetic distribution of everyday conversations by native Korean speakers. The nasal passage was

\section{H I}

- With age, nasal cavity volume increased, and nasal resistance decreased.

- Nasalance scores for the passages containing nasal consonants decreased with age.

- The age-related decreases in nasality may result from factors other than changes in the nasal cavity.
Table 1. Korean passages used for nasometric test

\begin{tabular}{|c|c|}
\hline Passage & International Phonetic Alphabet (IPA) \\
\hline \multicolumn{2}{|l|}{ Oral passage } \\
\hline $\begin{array}{l}\text { Devoid of nasal consonants, } \\
33 \text { syllables }\end{array}$ & $\begin{array}{l}\text { su.hi.ka.jo.gi.pa.da.e.kat.t'a.pe.do.tha. } \\
\text { go.thju.bi.do.tat.t'a.kap.ja.gi.pi:ga.wa.sə. } \\
\text { ji.bi.ro.to.la.wat.t'a./ }\end{array}$ \\
\hline \multicolumn{2}{|l|}{ Oral-nasal passage } \\
\hline $\begin{array}{l}32.6 \% \text { Nasal consonants, } \\
37 \text { syllables }\end{array}$ & 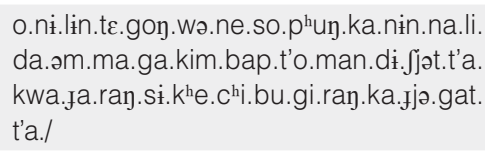 \\
\hline \multicolumn{2}{|l|}{ Nasal passage } \\
\hline $\begin{array}{l}\text { Devoid of oral consonants, } \\
24 \text { syllables }\end{array}$ & $\begin{array}{l}\text { əm.ma.an.njəy.əm.ma.an.njəy.me.mi. } \\
\text { an.njəy.me.mi.an.njəy.na.mu.an.njəy. } \\
\text { na.mu.an.njəy./ }\end{array}$ \\
\hline
\end{tabular}

devoid of oral consonants and was used to assess hyponasality (Table 1). Nasalance scores for each passage were measured three times and values for each subject were based on the averages of three measurements.

\section{Measurement of nasal cavity volume and nasal resistance}

On the same day, the nasal cavity volumes were measured using a RhinoScan 2.5 system (model SRE-2000; Interacoustics, Assens, Denmark). AR was performed separately for each nasal cavity according to the manufacturer's instructions. The measurements were accepted as suitable for evaluation when three repeatable area-distance curves were obtained. The endonasal volume of each nasal cavity, between the entrance to the nostril and $7 \mathrm{~cm}$ cephalically, was calculated, and the values were based on the average of three measurements derived from acceptable graphs. The total nasal volume was calculated by summing the values of both nasal cavities.

Active anterior rhinomanometry was performed to determine nasal resistance using a RhinoStream 2.0 system (model SRE2000, Interacoustics). Inspiratory and expiratory nasal resistances at a pressure of $150 \mathrm{~Pa}$ were measured on both sides according to the manufacturer's instructions. The total nasal resistance was calculated using the following formula: $R_{T}=R_{R} \times R_{L} /\left(R_{R}+R_{L}\right)$. $R_{T}$ means total nasal resistance; $R_{R}$, resistance of right side; $R_{L}$, resistance of left side.

\section{Statistical analysis}

Statistical analyses were performed using IBM SPSS ver. 22.0 (IBM Corp., Armonk, NY, USA) and GraphPad Prism 6 ver. 6.01 (GraphPad Software, La Jolla, CA, USA). The normality of distribution was determined using the D'Agostino-Pearson omnibus normality test. Differences among groups were analyzed with the Kruskal-Wallis test and Mann-Whitney $U$-test (twotailed). Nonparametric Spearman correlation coefficients were computed as indices of the strength of relationships between variables and age. In all analyses, $P<0.05$ was taken to indicate statistical significance. 


\section{RESULTS}

The nasal cavity volume, inspiratory and expiratory nasal resistance, and nasalance scores for all speech passages are summarized by age group in Table 2 . There were no significant sex differences in any of these variables (Table 3).

\section{Age-related changes in nasal cavity volume and nasal resistance}

The AR measurements demonstrated a progressive and relatively linear increase in nasal cavity volume with age. There was a strong positive correlation between age and nasal cavity volume, with a Spearman's $r$-value of $0.533(P<0.001)$ (Fig. 1A).

The total inspiratory nasal resistance (Fig. 1B) and total expiratory nasal resistance (Fig. 1C) of the total study population showed age-dependent decreases, with Spearman's $r$-values of
$-0.229(P=0.002)$ and $-0.231(P=0.002)$, respectively.

Age-related changes in nasalance score

Nasalance scores for the oronasal passage (Fig. 2A) and the na-

Table 3. Comparisons of measured data according to sex

\begin{tabular}{lrrr}
\hline Measurement & Male $(\mathrm{n}=90)$ & Female $(\mathrm{n}=90)$ & $P$-value \\
\hline Nasal cavity volume $\left(\mathrm{cm}^{3}\right)$ & $27.21 \pm 7.09$ & $26.24 \pm 6.84$ & 0.345 \\
Total nasal resistance $\left(\mathrm{Pa} / \mathrm{cm}^{3} / \mathrm{sec}\right)$ & $0.25 \pm 0.14$ & $0.28 \pm 0.16$ & 0.108 \\
$\quad$ Inspiratory & $0.26 \pm 0.14$ & $0.29 \pm 0.17$ & 0.066 \\
$\quad$ Expiratory & & & \\
Nasalance score $(\%)$ & $13.38 \pm 4.14$ & $14.21 \pm 4.90$ & 0.560 \\
$\quad$ Oral passage & $36.12 \pm 6.80$ & $36.17 \pm 7.32$ & 0.881 \\
Oronasal passage & $61.03 \pm 7.21$ & $60.44 \pm 6.25$ & 0.675 \\
\hline Nasal passage & & & \\
\hline
\end{tabular}

Values are presented as mean \pm standard deviation.

Table 2. Nasal cavity volume, nasal resistance, and nasalance according to age group

\begin{tabular}{|c|c|c|c|c|c|c|c|}
\hline \multirow{2}{*}{ Age (yr) } & \multirow{2}{*}{ Number } & \multirow{2}{*}{$\begin{array}{l}\text { Nasal cavity } \\
\text { volume }\left(\mathrm{cm}^{3}\right)\end{array}$} & \multicolumn{2}{|c|}{ Nasal resistance $\left(\mathrm{Pa} / \mathrm{cm}^{3} / \mathrm{sec}\right)$} & \multicolumn{3}{|c|}{ Nasalance score (\%) } \\
\hline & & & Inspiratory & Expiratory & Oral passage & Oronasal passage & Nasal passage \\
\hline $20-29$ & 36 & $22.26 \pm 5.16$ & $0.41 \pm 0.26$ & $0.39 \pm 0.25$ & $13.67 \pm 5.58$ & $39.58 \pm 6.29$ & $65.67 \pm 5.04$ \\
\hline 30-39 & 36 & $24.10 \pm 4.14$ & $0.24 \pm 0.11$ & $0.23 \pm 0.10$ & $14.36 \pm 5.66$ & $36.39 \pm 7.07$ & $59.94 \pm 6.38$ \\
\hline $40-49$ & 36 & $25.67 \pm 4.73$ & $0.26 \pm 0.09$ & $0.26 \pm 0.09$ & $13.42 \pm 3.52$ & $33.25 \pm 6.33$ & $57.56 \pm 6.31$ \\
\hline $50-59$ & 36 & $28.98 \pm 7.69$ & $0.24 \pm 0.09$ & $0.23 \pm 0.09$ & $13.53 \pm 4.47$ & $36.61 \pm 7.30$ & $61.50 \pm 6.98$ \\
\hline$\geq 60$ & 36 & $32.61 \pm 7.28$ & $0.22 \pm 0.08$ & $0.21 \pm 0.07$ & $14.00 \pm 3.07$ & $34.89 \pm 6.93$ & $59.03 \pm 6.12$ \\
\hline
\end{tabular}

Values are presented as mean \pm standard deviation.
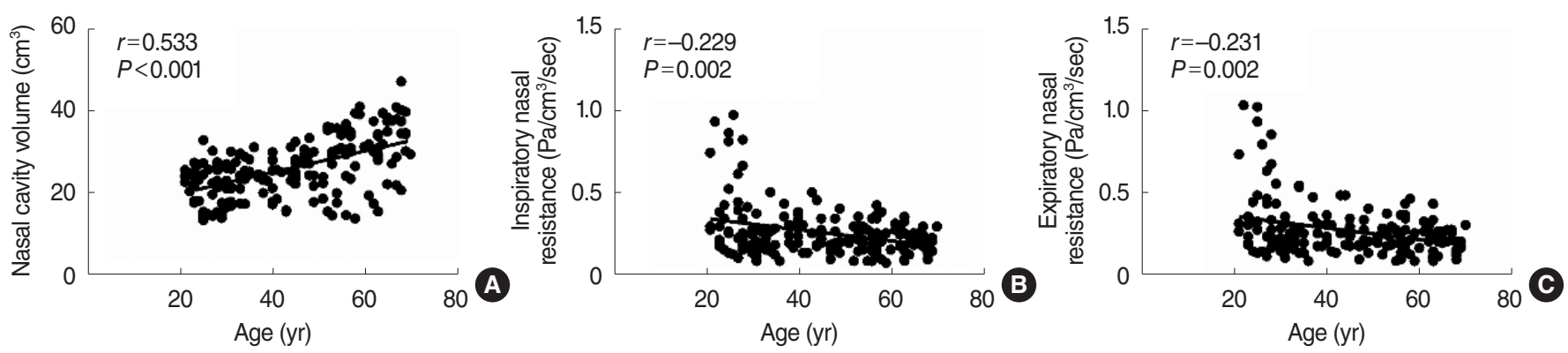

Fig. 1. Correlations of age with nasal cavity volume (A), total inspiratory nasal resistance (B), and total expiratory resistance (C). Data are shown as scatterplots with linear regression curves.
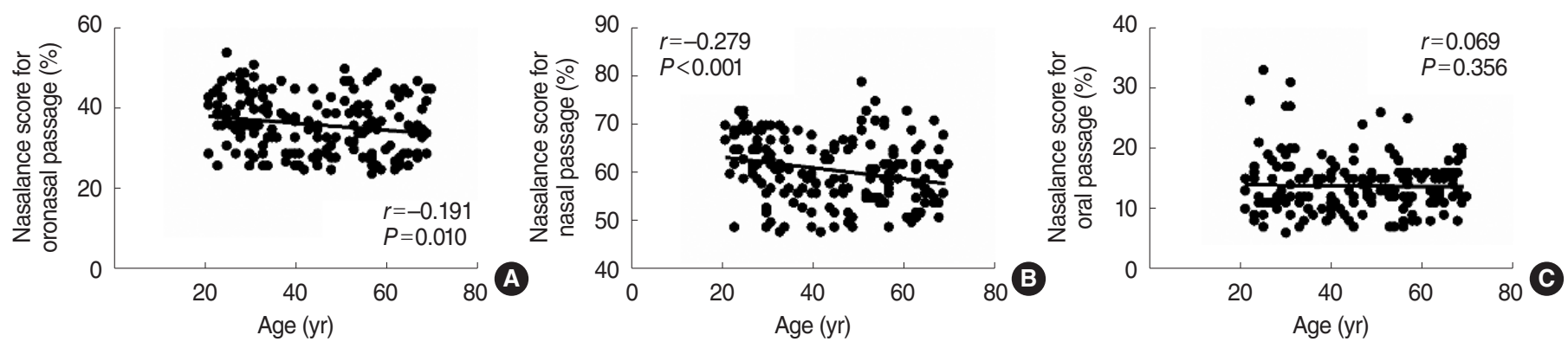

Fig. 2. Correlations of age and nasalance score for oronasal passage (A), nasal passage (B), and oral passage (C). Data are shown as scatterplots with linear regression curves. 
sal passage (Fig. 2B) decreased significantly with age $(r=-0.191$, $P=0.010$ and $r=-0.279, P<0.001$, respectively). However, there was no significant correlation between nasalance score for oral passage and age ( $r=0.069, P=0.356)$ (Fig. $2 \mathrm{C})$.

\section{DISCUSSION}

Normative data on nasalance scores in adults are needed to provide reference information for assessment of resonance in patients with resonance problems, and who may be candidates for sinonasal or velopharyngeal surgery. There have been a number of nasometric studies designed to obtain normative values for various languages. However, race, dialect, age, and sex have been suggested to affect nasalance scores [5,6]. All participants in the present study were native Korean speakers living in Daejeon city, to minimize linguistic and dialectal influences.

Sex has been suggested as a possible factor affecting nasalance scores, but there has been some controversy regarding this. Some studies reported no significant differences between sexes $[6,11]$, while many others showed that women have higher nasalance scores than men $[2,4,5,7,8]$. A number of studies showed sex differences in nasal cavity volume and intranasal cross-sectional area, which may affect nasalance scores $[12,13]$. However, the results of the present study indicated no sex differences in nasal cavity volume or nasal resistance when compared within each age group, as well as the whole-population level. Furthermore, there were no significant sex differences in nasalance scores for any of the passages tested.

Aging is a basic physiological process affecting structural and functional aspects of all parts of the body, including the nasal cavity and velopharynx. Therefore, age is also considered as a variable possibly influencing nasalance scores.

All structural components of the nose are known to undergo changes, including the skin, muscles, cartilage, bones, and nasal mucosa, with age [12]. Therefore, the nasal cavity dimensions and resistance may also be assumed to change with age. Indeed, Kalmovich et al. [12] utilized AR to measure the nasal geometry in a healthy adult population, and reported gradual increases in minimal cross-sectional area and nasal volume with age. Loftus et al. [13] also reported a progressive and relatively linear increase in intranasal volume with increasing age. However, there have been conflicting reports regarding the age-related changes in nasal resistance. Edelstein [14] reported that nasal resistance increased with age, whereas other groups reported that nasal resistance decreased with age $[15,16]$. In the present study, there was a progressive and relatively linear increase in nasal cavity volume with increasing age, while nasal resistance showed an age-dependent decrease. Comparison by age group revealed that the nasal resistance decreased markedly from the third to fourth decade of life.

The nasal cavity and related structures are known to affect nasalance. A number of studies demonstrated that increases in nasal cavity volume and decreases in nasal resistance result in increased nasalance [17-19]. Therefore, the progressive increase in nasal cavity volume and decrease in nasal resistance with age may be assumed to lead to increased resonance and higher nasalance scores. However, in contrast to our expectations, the present study showed that nasalance scores for either the oronasal passage or the nasal passage containing nasal consonants decreased significantly with age, while there was no significant correlation between nasalance score for the oral passage and age. Thus, factors other than changes in the nasal cavity are thought to play more critical roles in age-related changes in nasality.

The velopharynx is a complex structure that is responsible for separation of the oral and nasal cavities during speech production. The velopharyngeal sphincter is bounded anteriorly by the soft palate, laterally by the lateral pharyngeal wall, and posteriorly by the posterior pharyngeal wall [20]. The velopharyngeal port closes while vowels are formed, with the sound being generated in the oral cavity, but it is open during production of nasal consonants. Therefore, velopharyngeal function is another important factor related to changes in nasality.

A number of studies have demonstrated age-related changes in pharyngeal morphology and physiology [21-24]. Martin et al. [21] reported a decrease in upper airway size with increasing age. Johnston and Richardson [22] examined changes in pharyngeal morphology with age based on cephalometric measurements and found that the depth of the airway decreased, while the soft palate became longer and thicker, with age. Malhotra et al. [23] reported significant age-related increases in parapharyngeal fat pads and soft palate length, and changes in the bone shape surrounding the pharynx based on magnetic resonance imaging analysis. Guo et al. [24] also reported that elderly subjects had a larger soft palate fat pad volume compared to younger subjects. Therefore, it may be assumed that age-related morphological changes in the velopharyngeal port would reduce nasality during production of nasal consonants, as well as cause increased pharyngeal collapsibility during sleep.

In the present study, there was no significant correlation between nasalance score for oral passage without nasal consonants and age, whereas nasalance scores for either the oronasal passage or the nasal passage containing nasal consonants decreased significantly with age. Although we did not evaluate age-related changes in the velopharynx in this study, our results, and those of the abovementioned morphological studies, suggest that the opening function of the velopharyngeal sphincter decreases with age, thus resulting in a decrease in nasality during production of nasal consonants.

To our knowledge, this is the first study to investigate the effects of aging on nasality in normal healthy adults, and to investigate the influence of age-related changes in nasal cavity volume and nasal patency on nasality changes. This study demon- 
strated that nasalance scores for passages containing nasal consonants decreased significantly with age, despite significant increases in nasal cavity volume and nasal patency. Therefore, agerelated changes in the nasal cavity may not be the dominant factor affecting nasality. We postulated that velopharyngeal dysfunction with age may play a more critical role in the age-related reduction in nasality. To validate this hypothesis, further studies are needed to evaluate age-related changes in velopharyngeal biomechanics during the production of speech.

\section{CONFLICT OF INTEREST}

No potential conflict of interest relevant to this article was reported.

\section{REFERENCES}

1. Hong KH, Kwon SH, Jung SS. The assessment of nasality with a nasometer and sound spectrography in patients with nasal polyposis. Otolaryngol Head Neck Surg. 1997 Oct;117(4):343-8.

2. Park M, BaekWS, Lee E, Koh KS, Kim BK, Baek R. Nasalance scores for normal Korean-speaking adults and children. J Plast Reconstr Aesthet Surg. 2014 Feb;67(2):173-7.

3. Hosemann W, Gode U, Dunker JE, Eysholdt U. Influence of endoscopic sinus surgery on voice quality. Eur Arch Otorhinolaryngol. 1998;255(10):499-503.

4. Van Lierde KM, Wuyts FL, De Bodt M, Van Cauwenberge P. Age-related patterns of nasal resonance in normal Flemish children and young adults. Scand J Plast Reconstr Surg Hand Surg. 2003;37(6): 344-50.

5. Mishima K, Sugii A, Yamada T, Imura H, Sugahara T. Dialectal and gender differences in nasalance scores in a Japanese population. $\mathrm{J}$ Craniomaxillofac Surg. 2008 Jan;36(1):8-10.

6. Brunnegard K, van Doorn J. Normative data on nasalance scores for Swedish as measured on the Nasometer: influence of dialect, gender, and age. Clin Linguist Phon. 2009 Jan;23(1):58-69.

7. Abou-Elsaad T, Quriba A, Baz H, Elkassaby R. Standardization of nasometry for normal Egyptian Arabic speakers. Folia Phoniatr Logop. 2012;64(6):271-7.

8. Karakoc O, Akcam T, Birkent H, Arslan HH, Gerek M. Nasalance scores for normal-speaking Turkish population. J Craniofac Surg. 2013 Mar;24(2):520-2.

9. Awan SN, Bressmann T, Poburka B, Roy N, Sharp H, Watts C. Dialectical effects on nasalance: a multicenter, cross-continental study. J Speech Lang Hear Res. 2015 Feb;58(1):69-77.

10. Ha S, Cho SH. Nasalance scores for normal Korean-speaking adults and children: effects of age, vowel context, and stimulus length. Int J Pediatr Otorhinolaryngol. 2015 Aug;79(8):1235-9.

11. van Doorn J, Purcell A. Nasalance levels in the speech of normal Australian children. Cleft Palate Craniofac J. 1998 Jul;35(4):287-92.

12. Kalmovich LM, Elad D, Zaretsky U,Adunsky A, Chetrit A, Sadetzki $\mathrm{S}$, et al. Endonasal geometry changes in elderly people: acoustic rhinometry measurements. J Gerontol A Biol Sci Med Sci. 2005 Mar; 60(3):396-8.

13. Loftus PA, Wise SK, Nieto D, Panella N, Aiken A, DelGaudio JM. Intranasal volume increases with age: computed tomography volumetric analysis in adults. Laryngoscope. 2016 Oct;126(10):2212-5.

14. Edelstein DR. Aging of the normal nose in adults. Laryngoscope. 1996 Sep;106(9 Pt 2):1-25.

15. Kim SW, Mo JH, Kim JW, Kim DY, Rhee CS, Lee CH, et al. Change of nasal function with aging in Korean. Acta Otolaryngol Suppl. 2007 Oct;(558):90-4.

16. Vig PS, Zajac DJ. Age and gender effects on nasal respiratory function in normal subjects. Cleft Palate Craniofac J. 1993 May;30(3): 279-84.

17. Williams RG, Eccles R, Hutchings H. The relationship between nasalance and nasal resistance to airflow. Acta Otolaryngol. 1990 NovDec;110(5-6):443-9.

18. Birkent H, Erol U, Ciyiltepe M, Eadie TL, Durmaz A, Tosun F. Relationship between nasal cavity volume changes and nasalance. J Laryngol Otol. 2009 Apr;123(4):407-11.

19. Jiang RS, Huang HT. Changes in nasal resonance after functional endoscopic sinus surgery. Am J Rhinol. 2006 Jul-Aug;20(4):432-7.

20. Raol N, Hartnick CJ. Anatomy and physiology of velopharyngeal closure and insufficiency. Adv Otorhinolaryngol. 2015;76:1-6.

21. Martin SE, Mathur R, Marshall I, Douglas NJ. The effect of age, sex, obesity and posture on upper airway size. Eur Respir J. 1997 Sep; 10(9):2087-90.

22. Johnston $C D$, Richardson A. Cephalometric changes in adult pharyngeal morphology. Eur J Orthod. 1999 Aug;21(4):357-62.

23. Malhotra A, Huang Y, Fogel R, Lazic S, Pillar G, Jakab M, et al. Aging influences on pharyngeal anatomy and physiology: the predisposition to pharyngeal collapse. Am J Med. 2006 Jan;119(1):72.e9-14.

24. Guo J, Gao XM, Li XD, Zeng XL. Magnetic resonance imaging survey of the upper airway in different age non-snoring males. Zhonghua Er Bi Yan Hou Tou Jing Wai Ke Za Zhi. 2008 Sep;43(9):676-80. 